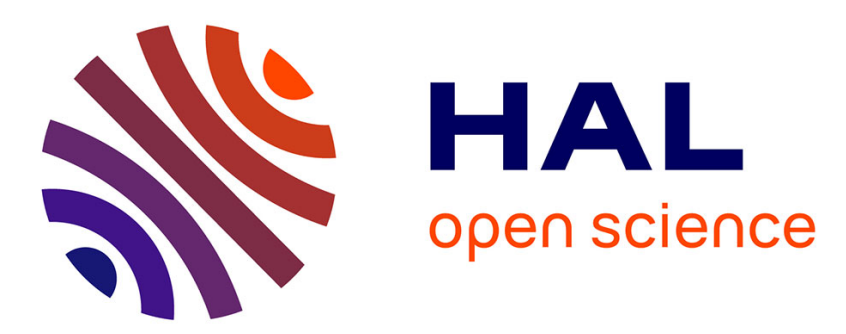

\title{
Thermal and thermoelastic behaviour of multiply coated inclusion-reinforced composites
}

\author{
E. Hervé
}

\section{To cite this version:}

E. Hervé. Thermal and thermoelastic behaviour of multiply coated inclusion-reinforced composites. International Journal of Solids and Structures, 2002, 39, pp.1041-1058. 10.1016/S0020-7683(01)002578. hal-00111351

\section{HAL Id: hal-00111351 \\ https://hal.science/hal-00111351}

Submitted on 27 Aug 2019

HAL is a multi-disciplinary open access archive for the deposit and dissemination of scientific research documents, whether they are published or not. The documents may come from teaching and research institutions in France or abroad, or from public or private research centers.
L'archive ouverte pluridisciplinaire HAL, est destinée au dépôt et à la diffusion de documents scientifiques de niveau recherche, publiés ou non, émanant des établissements d'enseignement et de recherche français ou étrangers, des laboratoires publics ou privés. 


\title{
Thermal and thermoelastic behaviour of multiply coated inclusion-reinforced composites
}

\author{
E. Herve ${ }^{a, b, *}$ \\ ${ }^{a}$ Laboratoire de Mécanique des Solides, Ecole Polytechnique, CNRS, UMR 7649, F-91128 Palaiseau Cedex, France \\ ${ }^{\mathrm{b}}$ Université de Versailles Saint-Quentin-en-Yvelines, 45 Avenue des Etats Unis, 78035 Versailles Cedex, France
}

\begin{abstract}
This work is devoted first to the derivation of the temperature field in an infinite medium constituted of an $n$-layered isotropic spherical inclusion, embedded in a matrix subjected to a uniform temperature gradient at infinity, under assumptions of no coupling between mechanical and thermal effects and of steady state conditions. These results lead to an estimate of the effective thermal conductivity coefficient of an $n$-layered inclusion-reinforced material. The second objective of this work is the derivation of the thermoelastic stress and strain fields in the same basic configuration but now, a coupling between stress and thermal properties is considered and the infinite matrix is stress free and subjected to a uniform change of temperature. These results and the solution of the same problem with a hydrostatic loading are used to estimate the effective thermal expansion coefficient and the specific heats for heterogeneous inclusion-reinforced materials. (c) 2002 Elsevier Science Ltd. All rights reserved.
\end{abstract}

Keywords: Thermal behaviour; Thermoelasticity; Micromechanical modelling; $n$-Layered inclusion-reinforced composites

\section{Introduction}

The purpose of the present paper is to use an appropriate theory of thermoelasticity to deduce an estimate for the effective thermal conductivity, the effective thermal expansion coefficient and the specific heats in terms of the constituent properties for composite materials with multiphase inclusions. Rosen (1970) has shown that suitable thermoelastic energy functions can be expressed in terms of macroscopic (or average) state variables and effective composite properties. The problem of relating effective mechanical properties of linear elastic multiply coated inclusion-reinforced materials to constituent properties has already been solved (Hervé and Zaoui, 1993). In contrast, little can be found on the effective thermal behaviour of this type of composite materials. Nevertheless, for materials with other microstructures, bounds on effective thermal expansion coefficients (Schapery, 1968; Rosen and Hashin, 1970) and on specific heats (Rosen and Hashin, 1970) of anisotropic composites having any number of anisotropic phases (Rosen and

\footnotetext{
${ }^{*}$ Address for Correspondence: Laboratoire de Mécanique des Solides, Ecole Polytechnique, CNRS, UMR 7649, F-91128 Palaiseau Cedex, France. Fax: +33-1-39-25-30-15.

E-mail address: herve@lms.polytechnique.fr (E. Herve).
} 
Hashin, 1970) or having two or three isotropic phases (Schapery, 1968) have been derived by employing extremum principles of thermoelasticity. However, in these papers, the connectedness of the matrix surrounding the multiply layered inclusion cannot be taken into account. The exception is the work of Stolz (1999) where the Hashin-Shtrikman bounding method is extended to the case of thermoelastic heterogeneous medium defined by representative morphological patterns. Note that Christensen (1979) takes also into account the connectedness of the matrix but determines only the thermal conductivity and the thermal expansion coefficient of two-phase materials.

The present paper extends the " $(n+1)$-phase” model, already used in elasticity (Hervé and Zaoui, 1993, 1995a,b; Hervé et al., 1993; Hervé and Pellégrini, 1995), in viscoelasticity (Beurthey, 2000; Fen-Chong et al., 1999; Alberola and Mele, 1994, 1997; Alberola and Benzarti, 1997, 1998), in viscoplasticity (Hervé et al., 1995) and in elastoplasticity (Thébaud et al., 1992; Bornert et al., 1993, 1994), to thermal and thermoelastic behaviours. The main results are estimates of the thermal conductivity, of the thermal expansion coefficient and of the specific heats of multiply coated inclusion-reinforced composites.

In the following the studied configuration is composed of an $n$-layered isotropic spherical inclusion surrounded by an infinite matrix ( $n$ is arbitrary). An explicit solution of the temperature field (Section 2) is first proposed if a uniform temperature gradient is applied at infinity and no coupling is assumed between mechanical and thermal effects. This solution is used for the definition of a $(n+1)$-phase model providing an estimate of the effective thermal conductivity. Then, the coupling between stress and thermal properties is taken into account. We derive in Section 3 the thermoelastic stress and strain fields in such a configuration, stress free and subjected to a uniform change of temperature. These results, completed by the stress and strain fields for the same configuration subjected to a hydrostatic pressure (Hervé and Zaoui, 1993) are used to construct a $(n+1)$-phase model to predict the effective thermal expansion coefficient and the effective specific heats of multiply coated inclusion-reinforced composites. The effective thermal conductivity, thermal expansion coefficient and specific heats are proved to be the coincident bounds for an isotropic Hashin $n$-layered composite sphere assemblage ( $n$-CSA) and can thus be derived by a recursive algorithm (Stolz, 1999).

\section{Thermal conductivity}

\section{1. $n$-Layered spherical inclusion problem}

This section is concerned with the derivation, under assumptions of no coupling between mechanical and thermal effects and of steady state conditions, of the temperature field in an infinite medium constituted of an $n$-layered spherical inclusion, embedded in a matrix subjected to a uniform temperature rise at infinity. For that purpose we define:

$$
\theta=T-T_{0},
$$

where $T_{0}$ is the base temperature and $\|\overrightarrow{\operatorname{grad}} \theta\| \ll 1$. Each phase is assumed homogeneous and isotropic.

As in Hervé and Zaoui (1993) let us consider the following infinite medium. Phase 1 constitutes the central core and phase (i) lies within the shell limited by the two concentric spheres with the radii $R_{i-1}$ and $R_{i}$. Let $\left(\lambda=\lambda_{i} \mathbf{I}\right)$ be the thermal conductivity tensor of phase (i) (here $i \in[1, n+1], R_{0}=0$ and $R_{n+1} \rightarrow \infty$ ) (Fig. 1), in which $\mathbf{I}$ is the second order unit tensor.

Spherical coordinate system $(r, \theta, \phi)$ with origin at the common centre of the above-mentioned spheres is introduced with axial symmetry around $x_{3}$-axis $\left(x_{3}=r \cos \theta\right)$.

At infinity, the imposed condition is (Christensen, 1979):

$$
\left.\theta\right|_{r \rightarrow \infty}=\beta x_{3}
$$

where $x_{k}$ are Cartesian coordinates and $\beta$ is the temperature gradient $(\beta \ll 1)$. 


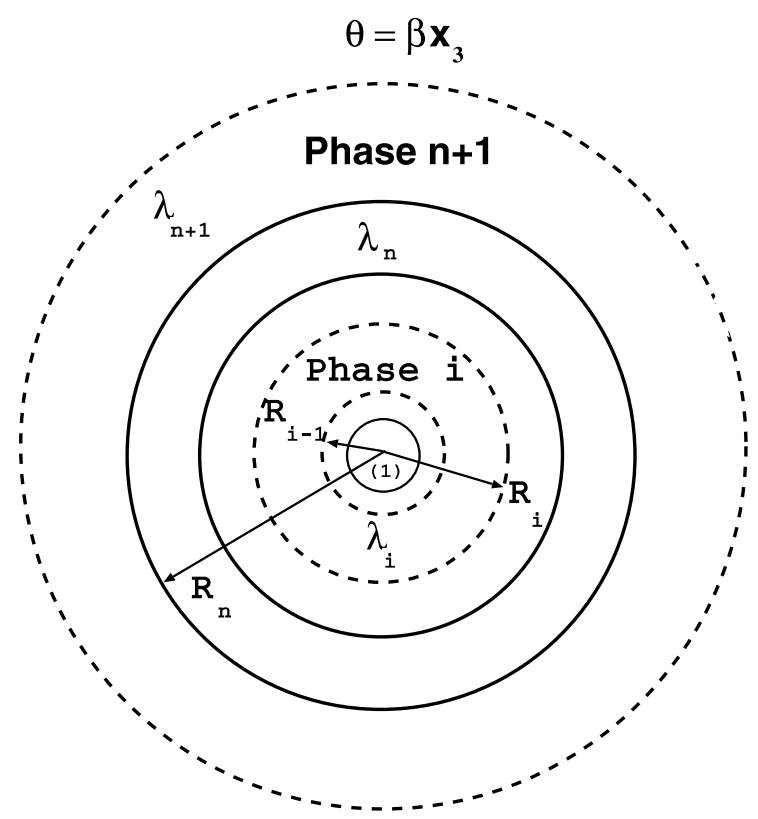

Fig. 1. The $n$-layered spherical inclusion embedded in an infinite matrix.

The assumption $\|\overrightarrow{\operatorname{grad}} \theta\| \ll 1$ permits the of use Fourier's law of heat conduction

$$
\vec{q}=-\lambda \overrightarrow{\operatorname{grad}} \theta
$$

where $\vec{q}$ represents the heat flux vector. With the additional assumption that there is no heat supply, the heat conduction equation writes

$$
\operatorname{div} \vec{q}=0 .
$$

From relations (3) and (4) we get the following governing equations for $\theta^{(k)}$ in each phase $k$

$$
\Delta \theta^{(k)}=0, \quad k \in[1, n+1],
$$

where the $\Delta$ operator in the spherical coordinate system reads:

$$
\Delta=\frac{1}{r^{2}} \frac{\partial}{\partial r}\left(r^{2} \frac{\partial}{\partial r}\right)+\frac{1}{r^{2} \sin \theta} \frac{\partial}{\partial \theta}\left(\sin \theta \frac{\partial}{\partial \theta}\right) .
$$

Thus, the general solution of Eq. (5), for the non-zero temperature rise $\theta^{(i)}$, which respects the remote condition (2), is given in phase (i) (Christensen, 1979) by

$$
\theta^{(i)}=\left(A_{i} r+\frac{B_{i}}{r^{2}}\right) \cos \theta
$$

where $A_{i}$ and $B_{i}$ are constants. The corresponding heat flux vector has for components:

$$
q_{r}^{(i)}=-\lambda_{i}\left(A_{i}-2 \frac{B_{i}}{r^{3}}\right) \cos \theta, \quad q_{\theta}^{(i)}=\lambda_{i}\left(A_{i}+\frac{B_{i}}{r^{3}}\right) \sin \theta, \quad q_{\phi}^{(i)}=0,
$$


where the coefficient $B_{1}$ must vanish to avoid a singularity at the origin and the constant $A_{n+1}$ is determined by the imposed temperature rise at infinity:

$$
\theta^{(n+1)} \rightarrow A_{n+1} x_{3} \quad \text { as } r \rightarrow \infty .
$$

Using the condition (2) and relation (9) the following value for $A_{n+1}$ is obtained

$$
A_{n+1}=\beta .
$$

It is worth noticing that the imposed condition at infinity can also be of a flux-type

$$
\vec{q}=-\gamma \vec{e}_{3}
$$

and, in that case, we find

$$
A_{n+1}=\frac{\gamma}{\lambda_{n+1}} \text {. }
$$

The two conditions that result from the continuity of $q_{r}$ and $\theta$ at the interface $r=R_{k}$ between phases $(k)$ and $(k+1)$ are written in the form

$$
\mathbf{H}_{k}\left(R_{k}\right) \vec{V}_{k}=\mathbf{H}_{k+1}\left(R_{k}\right) \vec{V}_{k+1}
$$

where $k \in[1, n], \vec{V}_{k}=\left(A_{k}, B_{k}\right)$ and $\mathbf{H}_{k}$ is the following matrix

$$
\mathbf{H}_{k}(r)=\left(\begin{array}{cc}
r & \frac{1}{r^{2}} \\
\lambda_{k} & -2 \frac{\lambda_{k}}{r^{3}}
\end{array}\right) \text {. }
$$

The system of equation (13) is solved for $\vec{V}_{k+1}$ by means of the "transfer matrices" I $\mathbf{I}^{(k)}$

$$
\vec{V}_{k+1}=\mathbf{I}^{(k)} \vec{V}_{k}
$$

with

$$
\mathbf{I}^{(k)}=\mathbf{H}_{k+1}^{-1}\left(R_{k}\right) \mathbf{H}_{k}\left(R_{k}\right) .
$$

From Eqs. (14) and (16), $\mathbf{I}^{(k)}$ reads

$$
\left.\mathbf{I}^{(k)}=\frac{1}{3 \lambda_{k+1}} \quad \begin{array}{ccc}
2 \lambda_{k+1}+\lambda_{k} & \frac{2}{R_{k}^{3}}\left(\lambda_{k+1}-\lambda_{k}\right) \\
R_{k}^{3}\left(\lambda_{k+1}-\lambda_{k}\right) & 2 \lambda_{k}+\lambda_{k+1}
\end{array}\right) .
$$

Therefore one gets

$$
\vec{V}_{k+1}=\prod_{j=k}^{1} \mathbf{I}^{(j)} \vec{V}_{1}=\mathbf{O}^{(k)} \vec{V}_{1},
$$

and, from Eq. (18) with $B_{1}=0$ we find

$$
A_{1}=\frac{1}{O_{11}^{(n)}} A_{n+1} .
$$

Thus all the coefficients $A_{k}$ and $B_{k}$ can be expressed as:

$$
\left\{\begin{array}{l}
A_{k}=\frac{O_{11}^{(k-1)}}{O_{11}^{(n)}} A_{n+1} \quad k \in[1, n], \\
B_{k}=\frac{O_{21}^{(k-1)}}{O_{11}^{(n)}} A_{n+1} .
\end{array}\right.
$$




\subsection{Average temperature gradient and heat flux vector}

From the solution derived in the previous section, the third component of the average temperature gradient in each phase is easily calculated with the relation

$$
\left\langle\theta_{, 3}^{(k)}\right\rangle=\frac{1}{V_{k}} \int_{V_{k}} \theta_{, 3}^{(k)} \mathrm{d} V=\frac{1}{V_{k}}\left[\int_{S_{k}} \theta^{(k)} n_{3} \mathrm{~d} S-\int_{S_{k-1}} \theta^{(k)} n_{3} \mathrm{~d} S\right] .
$$

In Eq. (21) $V_{k}$ is the volume of the shell occupied by phase $(k)$, limited by $S_{k-1}$ and $S_{k}$ and $n_{3}\left(n_{3}=\cos \theta\right)$ denotes the third component of the unit outward normal to $S_{k-1}$ or $S_{k}$ in a cartesian coordinate system $\left(S_{0}\right.$ is the point $r=0$ and $\left.S_{n+1} \rightarrow \infty\right)$.

The average value $\left\langle\theta_{, 3}^{(k)}\right\rangle$ is given by

$$
\left\langle\theta_{, 3}^{(k)}\right\rangle=A_{k},
$$

if condition (2) or condition (11) is prescribed at infinity. This average is also calculated in the whole $n$ layered inclusion

$$
\left\langle\theta_{, 3}\right\rangle=\left(A_{n+1}+\frac{B_{n+1}}{R_{n}^{3}}\right) .
$$

Correspondingly, still with condition (2) or condition (11), the third component of the average of the heat flux vector reads

$$
\left\langle q_{3}^{(k)}\right\rangle=-\lambda_{k} A_{k},
$$

and is given in the whole $n$-layered inclusion by

$$
\left\langle q_{3}\right\rangle=-\lambda_{n+1}\left[\left(A_{n+1}+\frac{B_{n+1}}{R_{n}^{3}}\right)\right] .
$$

These solutions can be used if a true $n$-phase inclusion is considered in a well defined matrix whose thermal conductivity coefficient $\lambda_{n+1}$ is known. It can also be used if $\lambda_{n+1}$ (named then $\lambda^{\text {eff }}$ ) denotes the effective thermal conductivity coefficient of a composite material according to the $(n+1)$-phase model presented in the next section.

\section{3. $A(n+1)$-phase model}

To determine the effective thermal conductivity coefficient of an isotropic composite material the " $(n+1)$-phase model" is defined as follows: if condition (2) is imposed, the third component of the average temperature gradient in the whole $n$-layered inclusion is the same as the third component of the macroscopic temperature gradient imposed to the composite medium at infinite $\left(\left\langle\theta_{, 3}\right\rangle=\beta\right)$. From Eq. (23) this condition reduces to

$$
B_{n+1}=0 .
$$

Note that a similar self-consistent condition could be proposed from Eq. (25) and boundary condition (11) for the heat flux vector.

Let us introduce the virtual work $Q$ (Hashin, 1972):

$$
Q=-\iiint_{V} \vec{q} \cdot \overrightarrow{\operatorname{grad}} \theta \mathrm{d} v
$$

$Q_{\text {comp }}$ and $Q_{0}$ are the virtual works calculated over the composite configuration shown in Fig. 1 and over a second configuration. This second configuration is a body identical to the first one except that the inclusion 
is replaced by the equivalent homogeneous material characterized by $\lambda^{\text {eff }}$. Following the same treatment as the one used in (Christensen, 1979) for the elastic energy we can show that:

$$
Q_{\text {comp }}=Q_{0} \pm \iint_{S_{n}}\left(\overrightarrow{q^{0}} \theta-\vec{q} \theta^{0}\right) \cdot \vec{n} \mathrm{~d} S
$$

where $\overrightarrow{q^{0}}$ and $\theta^{0}$ designate the field variables calculated in the homogeneous configuration and $\vec{q}, \theta$ the ones calculated in the composite configuration. $S_{n}$ denotes the surface of the composite inclusion and the sign in front of the integral in Eq. (28) depends on the conditions specified on the outer boundary (imposed heat flux vector or imposed temperature). Combining Eqs. (7) and (8) in the self-consistent condition $Q_{\text {comp }}=Q_{0}$ yields also relation (26).

The effective thermal conductivity, $\lambda^{\text {eff }}$, contributes only to the matrix $I^{(n)}$ so that relation (18) is modified to read

$$
\vec{V}_{n+1}=\mathbf{I}^{(n)} \prod_{j=n-1}^{1} \mathbf{I}^{(j)} \vec{V}_{1}=\mathbf{I}^{(n)} \mathbf{O}^{(n-1)} \vec{V}_{1} .
$$

Relation (17) is now substituted in Eq. (29) for $I^{(n)}$ with $\lambda_{n+1}=\lambda^{\text {eff }}$ so that $B_{n+1}$ reads

$$
B_{n+1}=\frac{1}{3 \lambda}\left[R_{n}^{3}\left(\lambda^{\text {eff }}-\lambda_{n}\right) O_{11}^{(n-1)}+\left(2 \lambda_{n}+\lambda^{\text {eff }}\right) O_{21}^{(n-1)}\right]
$$

Setting $B_{n+1}$ to zero provides the effective thermal conductivity coefficient

$$
\lambda^{\text {eff }}=\frac{\lambda_{n}\left[O_{11}^{(n-1)} R_{n}^{3}-2 O_{21}^{(n-1)}\right]}{R_{n}^{3} O_{11}^{(n-1)}+O_{21}^{(n-1)}} .
$$

A recursive algorithm could be invoked to obtain the same result. $\lambda_{(i)}^{\text {eff }}$ denotes the effective thermal conductivity coefficient associated with a $(i+1)$-phase model. Then the following relation relates $\lambda_{(i)}^{\text {eff }}$ and $\lambda_{(i-1)}^{\text {eff }}$

$$
\lambda_{(i)}^{\text {eff }}=\lambda_{i}+\frac{\lambda_{i} \frac{R_{i-1}^{3}}{R_{i}^{3}}}{\frac{\lambda_{i}}{\lambda_{(i-1)}^{\text {eff }}-\lambda_{i}}+\frac{1}{3}\left(\frac{R_{i}^{3}-R_{i-1}^{3}}{R_{i}^{3}}\right)} .
$$

If $i=2$ this equation (with $\lambda_{(1)}^{\text {eff }}=\lambda_{1}$ ) yields the effective conductivity coefficient which was obtained by Hashin and Shtrikman (1962). It is the coincident bounds of a CSA with the volume fraction $R_{1}^{3} / R_{2}^{3}$. The recursive algorithm can be summarized as follows: any "two-phase inner spheres" in the $n$-layered CSA, is replaced by a homogeneous one with the coefficient $\lambda_{(2)}^{\text {eff }}$. Second, the three-layered CSA is considered as a two-layered one with the coefficient $\lambda_{(2)}^{\text {eff }}$ in the central core with the volume fraction $R_{2}^{3} / R_{3}^{3}$ and $\lambda_{3}$ in the external layer within $R_{2}$ and $R_{3}$. These two steps are repeated until the increment $i$ reaches the number $n$ of phases considered in the CSA. The same results are applicable to electrical conductivity, magnetic permeability, dielectric constant and diffusivity values.

\section{Thermal expansion coefficient and specific heats}

\section{1. n-Layered spherical inclusion problem}

\subsubsection{Determination of the strain and stress fields}

Let us consider the problem of an infinite medium constituted of a $n$-layered isotropic spherical inclusion, embedded in a stress-free matrix subjected to a prescribed temperature $\theta=\theta_{0}$ as shown in Fig. 1, with 
coupling between stress and thermal properties. This section is concerned with the derivation of the temperature and of the displacement fields for all point of this configuration. Under steady state conditions, assuming that there is no heat supply, the heat conduction law takes the simple form

$$
\Delta \theta^{(i)}=0, \quad i \in[1, n+1],
$$

where $\theta^{(i)}$ denotes the temperature rise in phase $(i)$. Let $\left(\mu_{i}, k_{i}, \alpha_{i}\right)$ be respectively the shear modulus, the bulk modulus and thermal expansion coefficient of phase $(i)$. The equilibrium equation in phase $(i)$ reads

$$
\frac{3 k_{i}+\mu_{i}}{3} \overrightarrow{\operatorname{grad}}(\operatorname{div} \vec{u})+\mu_{i} \vec{\nabla} \vec{u}-3 k_{i} \alpha_{i} \overrightarrow{\operatorname{grad}} \theta^{(i)}=0 .
$$

The problem has spherical symmetry and is thus one-dimensional. The solution, for the non-zero temperature field $\theta^{(i)}$ of Eq. (34) is given in phase $(i)$ by

$$
\theta^{(i)}(r)=\left(C_{i}+\frac{D_{i}}{r}\right)
$$

where the coefficient $D_{1}$ must vanish to avoid the singularity at the origin. The constant $C_{n+1}$ is determined by the prescribed condition at infinity:

$$
C_{n+1}=\theta_{0} .
$$

The interface conditions require the continuity of the temperature $\theta$ and of the normal heat flux $\vec{q} \cdot \vec{n}, \vec{n}$ denotes the normal vector at the interface. It follows that

$$
D_{i}=0 \quad \text { and } \quad C_{i}=C_{n+1}=\theta_{0} \quad \forall i \in[1, n+1]
$$

and therefore

$$
\theta(r)=\theta_{0} \quad \forall r .
$$

Now, combining Eqs. (34) and (38) and using the spherical symmetry, one obtains

$$
\operatorname{div} \vec{u}=0
$$

which admits for solution for the non-zero displacement component $u_{r}^{(i)}$

$$
u_{r}^{(i)}=F_{i} r+\frac{G_{i}}{r^{2}} .
$$

Then, the corresponding stresses are found to be

$$
\begin{aligned}
& \sigma_{r r}^{(i)}=3 k_{i} F_{i}-\frac{4 \mu_{i}}{r^{3}} G_{i}-3 k_{i} \alpha_{i} \theta_{0}, \\
& \sigma_{\theta \theta}^{(i)}=\sigma_{\phi \phi}^{(i)}=3 k_{i} F_{i}+\frac{2 \mu_{i}}{r^{3}} G_{i}-3 k_{i} \alpha_{i} \theta_{0},
\end{aligned}
$$

while all other stress components vanish. Let us set $\vec{V}_{k}=\left(F_{k}, G_{k}\right), k \in(1, n)$; the continuity conditions for $\sigma_{r r}$ and $u_{r}$ at the interface $r=R_{k}$ are written in the form

$$
\mathbf{J}_{k}\left(R_{k}\right) \vec{V}_{k}+\theta_{0} \vec{L}_{k}=\mathbf{J}_{k+1}\left(R_{k}\right) \vec{V}_{k+1}+\theta_{0} \vec{L}_{k+1},
$$

where $\vec{L}_{k}=\left(0,-3 k_{k} \alpha_{k}\right)(k \in[1, n])$ and $\mathbf{J}_{i}$ is the following matrix (Hervé and Zaoui, 1993)

$$
\mathbf{J}_{i}(r)=\left(\begin{array}{cc}
r & \frac{1}{r^{2}} \\
3 k_{i} & -4 \frac{\mu_{i}}{r^{3}}
\end{array}\right) .
$$

The solution of the system of equation (42) is 


$$
\vec{V}_{k+1}=\mathbf{N}^{(k)} \vec{V}_{k}+\theta_{0} \mathbf{J}_{k+1}^{-1}\left(R_{k}\right)\left(\vec{L}_{k}-\vec{L}_{k+1}\right),
$$

where $\mathbf{N}^{(k)}$ is given by

$$
\mathbf{N}^{(k)}=\mathbf{J}_{k+1}^{-1}\left(R_{k}\right) \mathbf{J}_{k}\left(R_{k}\right) .
$$

Substituting for $J_{i}(r)$ from Eq. (43) into Eq. (45) yields

$$
\left.\mathbf{N}^{(k)}=\frac{1}{3 k_{k+1}+4 \mu_{k+1}} \quad \begin{array}{cc}
3 k_{k}+4 \mu_{k+1} & \frac{4}{R_{k}^{3}}\left(\mu_{k+1}-\mu_{k}\right) \\
3\left(k_{k+1}-k_{k}\right) R_{k}^{3} & 3 k_{k+1}+4 \mu_{k}
\end{array}\right) .
$$

Therefore one gets

$$
\vec{V}_{k+1}=\mathbf{Q}^{(k)} \vec{V}_{1}+\theta_{0} \mathbf{M}^{(k+1)}=\prod_{j=k}^{1} \mathbf{N}^{(j)} \vec{V}_{1}+\theta_{0} \vec{M}^{(k+1)},
$$

with

$$
\left.\vec{M}^{(k+1)}=\frac{3\left(k_{k+1} \alpha_{k+1}-k_{k} \alpha_{k}\right)}{3 k_{k+1}+4 \mu_{k+1}}\left(\begin{array}{c}
1 \\
-R_{k}^{3}
\end{array}\right)+\sum_{i=1}^{k-1} \frac{3\left(k_{i+1} \alpha_{i+1}-k_{i} \alpha_{i}\right)}{3 k_{i+1}+4 \mu_{i+1}} \prod_{j=k}^{i+1} \mathbf{N}^{(j)}\right)\left(\begin{array}{c}
1 \\
-R_{i}^{3}
\end{array}\right),
$$

with $k \in(1, n), \vec{M}^{(1)}=\overrightarrow{0}$ and $\mathbf{Q}^{(0)}=\mathbf{I}$.

Making use of relation (47), with $G_{1}=0$, we find

$$
F_{1}=\frac{F_{n+1}-\theta_{0} M_{1}^{(n+1)}}{Q_{11}^{(n)}} .
$$

Thus all the coefficients $F_{k}, G_{k}$ are

$$
\left\{\begin{array}{l}
F_{k}=\frac{Q_{1(1)}^{(k-1)}}{Q_{11}^{(n)}} F_{n+1}+\frac{\theta_{0}}{Q_{11}^{(n)}}\left[M_{1}^{(k)} Q_{11}^{(n)}-M_{1}^{(n+1)} Q_{11}^{(k-1)}\right] \quad k \in[1, n], \\
G_{k}=\frac{Q_{21}^{(k-1)}}{Q_{11}^{(n)}} F_{n+1}+\frac{\theta_{0}}{Q_{11}^{(n)}}\left[M_{2}^{(k)} Q_{11}^{(n)}-M_{1}^{(n+1)} Q_{21}^{(k-1)}\right] .
\end{array}\right.
$$

The infinite matrix being stress free $\left(\sigma_{r r}=0\right.$ at infinity), it results from Eq. (41) that

$$
F_{n+1}=\alpha_{n+1} \theta_{0} \text {. }
$$

Then, from Eqs. (50) and (51)

$$
\left\{\begin{array}{l}
F_{k}=\frac{\theta_{0}}{Q_{11}^{(n)}}\left[\alpha_{n+1} Q_{11}^{(k-1)}+M_{1}^{(k)} Q_{11}^{(n)}-M_{1}^{(n+1)} Q_{11}^{(k-1)}\right] \quad k \in[1, n] \\
G_{k}=\frac{\theta_{0}}{Q_{11}^{(n)}}\left[\alpha_{n+1} Q_{21}^{(k-1)}+M_{2}^{(k)} Q_{11}^{(n)}-M_{1}^{(n+1)} Q_{21}^{(k-1)}\right] .
\end{array}\right.
$$

Consequently, from Eqs. (40), (41) and (52) the displacement field $\vec{u}^{(i)}$ and the corresponding stresses are calculated for a prescribed temperature rise at infinity.

\subsubsection{Average strains and stresses}

From these solutions, the average strain and stress tensor in each phase are now calculated following (Hervé and Zaoui, 1993). The average strain tensor in phase $(i)$ is given by

$$
\left\langle\epsilon^{(i)}\right\rangle=F_{i}\left[\vec{e}_{1} \otimes \vec{e}_{1}+\vec{e}_{2} \otimes \vec{e}_{2}+\vec{e}_{3} \otimes \vec{e}_{3}\right]
$$

where $\vec{e}_{1}, \vec{e}_{2}$ and $\vec{e}_{3}$ denote the unit vectors if the configuration under study is referred to a cartesiancoordinate system. We obtain therefrom the average strain in the whole $n$-layered inclusion 


$$
\langle\epsilon\rangle=\left(F_{n+1}+\frac{G_{n+1}}{R_{n}^{3}}\right)\left[\vec{e}_{1} \otimes \vec{e}_{1}+\vec{e}_{2} \otimes \vec{e}_{2}+\vec{e}_{3} \otimes \vec{e}_{3}\right]=\left(\alpha_{n+1} \theta_{0}+\frac{G_{n+1}}{R_{n}^{3}}\right)\left[\vec{e}_{1} \otimes \vec{e}_{1}+\vec{e}_{2} \otimes \vec{e}_{2}+\vec{e}_{3} \otimes \vec{e}_{3}\right]
$$

Similarly, the average stress tensor in phase $(i)$ is given by

$$
\left\langle\boldsymbol{\sigma}^{(i)}\right\rangle=3 k_{i}\left(F_{i}-\alpha_{i} \theta_{0}\right)\left[\vec{e}_{1} \otimes \vec{e}_{1}+\vec{e}_{2} \otimes \vec{e}_{2}+\vec{e}_{3} \otimes \vec{e}_{3}\right],
$$

and it follows that the average stress in the whole $n$-layered inclusion, according to Eq. (51), takes the final form

$$
\langle\boldsymbol{\sigma}\rangle=-4 \frac{\mu_{n+1}}{R_{n}^{3}} G_{n+1}
$$

\subsection{Thermal expansion coefficient}

In order to determine the thermal expansion coefficient of a multiply coated inclusion-reinforced composite, let us consider the problem described in Section 2.1 but now the infinite matrix is both subjected to uniform stress conditions at infinity $\left(\overrightarrow{T_{0}}=\boldsymbol{\Sigma} \vec{n}\right)$ and to a prescribed temperature $\left(\theta=\theta_{0}\right)$ (Fig. 2$)$. We can split this problem $(P)$ into two elementary problems $\left(P^{\prime}\right)$ and $\left(P^{r}\right)$ which have been studied in Hervé and Zaoui (1993) and in Section 3.1 respectively.

In this section, $\left(\mu_{n+1}, k_{n+1}, \boldsymbol{\alpha}_{n+1}\right)$ are called $\left(\mu^{\text {eff }}, k^{\text {eff }}, \boldsymbol{\alpha}^{\text {eff }}\right)$. These three scalars denote the effective shear modulus, bulk modulus and thermal expansion tensor according to the $(n+1)$-phase model defined in Hervé and Zaoui (1993) for $\mu^{\text {eff }}$ and $k^{\text {eff }}$ and hereafter for $\alpha^{\text {eff }}$.

(P)

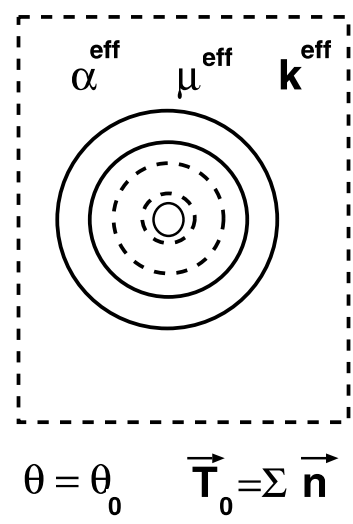

$\sigma(\mathbf{x})$
$\left(\mathbf{P}^{\prime}\right)$

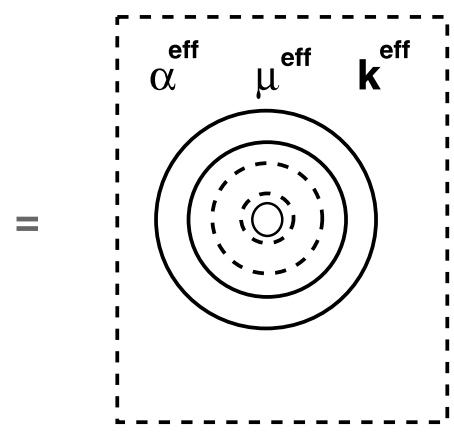

$\overrightarrow{\mathbf{T}}_{0}=\Sigma \overrightarrow{\mathbf{n}}$

$\theta=0$

$\sigma^{\prime}(\mathbf{x})$

$\varepsilon^{\prime}(\mathbf{x})$

$\varepsilon(\mathbf{x})$
$\left(\mathbf{P}^{\mathbf{r}}\right)$

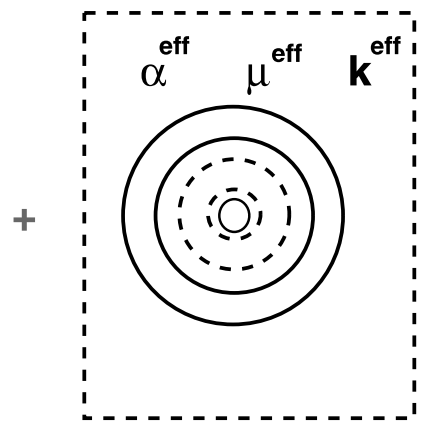

$\theta=\theta_{0}$

$\overrightarrow{\mathrm{T}}_{0}=\overrightarrow{\mathbf{0}}$

$\sigma(\mathbf{x})$

$\varepsilon(\mathbf{x})$

$\varepsilon(\mathbf{x})$

Fig. 2. Decomposition of the problem $(P)$ into two elementary problems $\left(P^{\prime}\right)$ and $\left(P^{r}\right)$. 
The stress field $\boldsymbol{\sigma}^{\prime}(x)$ is related to the prescribed stress $\boldsymbol{\Sigma}$ by the stress-concentration tensor $\mathbf{B}(x)$ which can be determined by means of the results of a previous paper (Hervé and Zaoui, 1993)

$$
\boldsymbol{\sigma}^{\prime}(x)=\mathbf{B}(x): \boldsymbol{\Sigma},
$$

where (:) stands for the second-order inner product $\left(\sigma: \epsilon=\sigma_{i j} \epsilon_{i j}\right)$. We define $\alpha^{\text {eff }}$ (according to the $(n+1)$ phase model) as the following link between the average strain $\mathbf{E}^{F}$ in the whole $n$-layered inclusion and the prescribed temperature

$$
\mathbf{E}^{F}=\left\langle\boldsymbol{\epsilon}^{r}(x)\right\rangle_{V_{\mathrm{I}}}=\boldsymbol{\alpha}^{\mathrm{eff}} \theta^{0} .
$$

This means that the composite inclusion behaves as the surrounding equivalent homogeneous medium. $V_{\mathrm{I}}$ denotes the volume of the $n$-layered inclusion. It follows from $\boldsymbol{\alpha}_{n+1}=\alpha^{\text {eff }}$ and from Eq. (54) that

$$
G_{n+1}=0 .
$$

Application of result (38) leads to the following expression

$$
\boldsymbol{\epsilon}^{r}(x)=\boldsymbol{\alpha}(x) \theta^{0}+\mathbf{s}: \boldsymbol{\sigma}^{r}(x),
$$

where $\mathbf{s}$ is the elastic compliance.

In Hervé and Zaoui (1993) it had been shown that

$$
\left\langle\boldsymbol{\sigma}^{\prime}\right\rangle_{V_{\mathrm{I}}}=\boldsymbol{\Sigma} \text {. }
$$

Therefore, accounting for Eq. (57), it can be inferred that

$$
\langle\mathbf{B}\rangle_{V_{\mathrm{I}}}=\mathbf{I},
$$

where I denotes the fourth order unit tensor.

The result given by Levin (1968) leads to the following relation:

$$
\boldsymbol{\alpha}^{\text {eff }}=\langle\boldsymbol{\alpha}: \mathbf{B}\rangle \text {. }
$$

Combining $\boldsymbol{\alpha}_{n+1}=\boldsymbol{\alpha}^{\text {eff }}$ and Eq. (62) yields

$$
\left\{\begin{array}{l}
\boldsymbol{\alpha}^{\text {eff }}=\lim _{V \rightarrow \infty}\left(\frac{V_{\mathrm{I}}}{V}\langle\boldsymbol{\alpha}: \mathbf{B}\rangle_{V_{\mathrm{I}}}+\frac{V-V_{\mathrm{I}}}{V} \boldsymbol{\alpha}^{\mathrm{eff}}:\langle\mathbf{B}\rangle_{V-V_{\mathrm{I}}}\right) \\
\boldsymbol{\alpha}^{\mathrm{eff}}=\lim _{V \rightarrow \infty}\left(\frac{V_{\mathrm{I}}}{V}\langle\boldsymbol{\alpha}: \mathbf{B}\rangle_{V_{\mathrm{I}}}+\frac{V-V_{\mathrm{I}}}{V} \boldsymbol{\alpha}^{\mathrm{eff}}\right),
\end{array}\right.
$$

and $\alpha^{\text {eff }}$ finally reduces to:

$$
\boldsymbol{\alpha}^{\mathrm{eff}}=\langle\boldsymbol{\alpha}: \mathbf{B}\rangle_{V_{1}} .
$$

Each phase is isotropic, therefore $\boldsymbol{\alpha}^{\mathrm{eff}}=\alpha^{\mathrm{eff}} \mathbf{I}, \boldsymbol{\alpha}(x)=\alpha(x) \mathbf{I}$ and

$$
\alpha^{\mathrm{eff}}=\frac{1}{3}\left\langle\alpha(x) B_{i i k k}\right\rangle_{V_{\mathrm{I}}} .
$$

It is worth noting that when $\boldsymbol{\Sigma}=\left(\sigma_{0} / 3\right) \mathbf{I}, B_{i k k k}(x)$ may be derived by means of

$$
\operatorname{Tr}(\boldsymbol{\sigma}(x))=B_{i i k k}(x) \frac{\sigma_{0}}{3}
$$

and then, by using Eqs. (15) and (34) and results in Hervé and Zaoui (1993) $B_{i i k k}$ is given in phase (l) by

$$
B_{i i k k}=\frac{3 Q_{11}^{(l-1)}}{Q_{11}^{(n)}} \frac{k_{l}}{k^{\mathrm{eff}}} .
$$

Consequently, the effective thermal expansion coefficient is provided by the relation 


$$
\alpha^{\mathrm{eff}}=\frac{1}{Q_{11}^{(n)} k^{\mathrm{eff}}} \sum_{i=1}^{n} c_{i} \alpha_{i} k_{i} Q_{11}^{(i-1)}
$$

where $c_{i}$ denotes the volume fraction of phase $(i)$.

As in the case of the thermal conductivity, it can be noticed that if $\alpha_{(i)}^{\text {eff }}$ denotes the effective thermal expansion coefficient associated with a $(i+1)$-phase model we get the following relation between $\alpha_{(n)}^{\text {eff }}$ and $\alpha_{(n-1)}^{\text {eff }}$

$$
\alpha_{(n)}^{\mathrm{eff}}=\frac{R_{n-1}^{3}}{R_{n}^{3}} \alpha_{(n-1)}^{\mathrm{eff}}+\left(1-\frac{R_{n-1}^{3}}{R_{n}^{3}}\right) \alpha_{n}+\cdots+\frac{4 \mu_{n} \frac{R_{n-1}^{3}}{R_{n}^{3}}\left(1-\frac{R_{n-1}^{3}}{R_{n}^{3}}\right)\left(k_{(n-1)}^{\mathrm{eff}}-k_{n}\right)^{2}}{\left(3 k_{n}+4 \mu_{n}\right) k_{(n-1)}^{\mathrm{eff}}+4 \mu_{n}\left(1-\frac{R_{n-1}^{3}}{R_{n}^{3}}\right)\left(k_{n}-k_{(n-1)}^{\mathrm{eff}}\right)}\left(\alpha_{(n-1)}^{\mathrm{eff}}-\alpha_{n}\right) .
$$

\subsection{Illustrative examples}

The above results allow us to better understand the problem of debonding along the two interfaces (interface inclusion/coated-phase here represented by $r=R_{1}$ and coated-phase/matrix here represented by $r=R_{2}$ ) of the interphase of a coated inclusion-reinforced composites. For that purpose Eqs. (41), (52) and (69) and the results for $k^{\text {eff }}$ and $\mu^{\text {eff }}$ known from Hervé and Zaoui (1993) are used to determine the normalized radial stress $\left(\sigma_{r r} / \theta_{0} k_{3} \alpha_{3}\right)$, along the two above-mentioned interfaces, due to an uniform temperature rise $\theta_{0}$. Fig. 3 gives this normalized stress in terms of the normalized bulk modulus $\left(k_{2} / k_{3}\right)$ of the interface. The analytical results show that these radial stresses depend linearly on the thermal expansion coefficient of the interphase $\left(\sigma_{r r}\left(R_{1}\right) / \theta_{0} k_{3} \alpha_{3}=a_{1} \alpha_{2} / \alpha_{3}+b_{1}\right.$ and $\left.\sigma_{r r}\left(R_{2}\right) / \theta_{0} k_{3} \alpha_{3}=a_{2} \alpha_{2} / \alpha_{3}+b_{2}\right)$. These four parameters are reported in Fig. $4 \mathrm{a}$ and $\mathrm{b}$ in terms of the normalized bulk modulus $\left(k_{2} / k_{3}\right)$ of the interphase. The illustrative examples (Figs. 3 and 4 ) prove that these models are easily tractable not only to predict the global behaviour of multiply coated inclusion-reinforced composites but also to determine some local major values, for instance here the sign of $\sigma_{r r}$ and so the debonding along the interfaces.

\subsection{Specific heats}

We now determine the effective specific heats for a multiply coated inclusion-reinforced composite. For that purpose, let us consider the configuration $\left(P^{r}\right)$ described in Fig. 2 where $C_{v_{i}}$ denotes the specific heat, per unit volume at constant volume in phase $(i)$ and $C_{v}^{\text {eff }}$ the effective specific heat of the infinite matrix according to the $(n+1)$-phase model defined hereafter.

For small strain and small temperature changes, the isotropic Helmholtz free energy density $F$ per unit volume is defined as (Rosen, 1970):

$$
F=\frac{1}{2} \epsilon: \mathbf{C}: \boldsymbol{\epsilon}+\boldsymbol{\Gamma}: \boldsymbol{\epsilon} \theta-\frac{1}{2} C_{v} \theta^{2},
$$

where $\mathbf{C}$ is the tensor of elastic moduli, $\boldsymbol{\epsilon}$ the strain tensor, $\boldsymbol{\Gamma}$ the thermal stress tensor, $\theta$ the temperature which are related to the stress tensor $\sigma$ and to the thermal expansion tensor $\boldsymbol{\alpha}$ by

$$
\epsilon=\mathbf{s}: \sigma+\alpha \theta
$$

and

$$
\Gamma=-\mathbf{C}: \boldsymbol{\alpha} .
$$

Note that in the definition of $F$ the term involving $C_{v}$ has a sign reversal from those in Rosen (1970), to be consistent with the classical thermodynamical derivations. $T_{0} C_{v} / \rho$ denotes here the specific heat at constant deformation. 


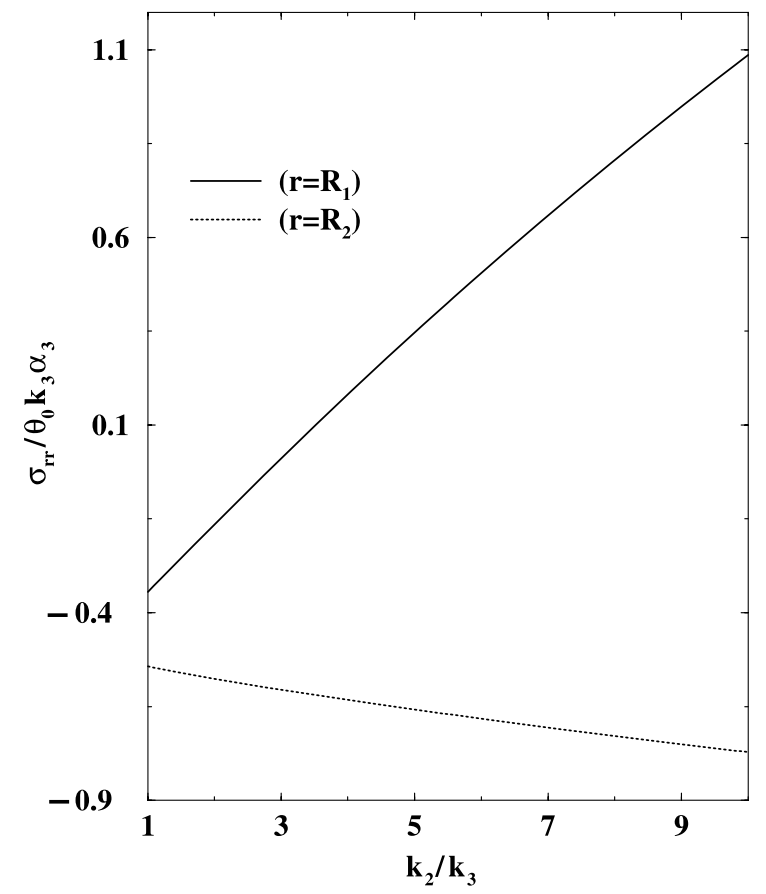

Fig. 3. Normalized radial stress $\sigma_{r r} / \theta_{0} k_{3} \alpha_{3}$ along the interface inclusion/coated-phase $\left(r=R_{1}\right)$ and along the interface coated-phase/ matrix here $\left(r=R_{2}\right)$ of the coated phase (phase 2) of a composite material made of coated inclusions versus the normalized bulk modulus $\left(k_{2} / k_{3}\right)$ of the coated phase with $k_{1} / k_{3}=2$. The Young moduli in the different phases are $v_{1}=0.17, v_{2}=v_{3}=0.3$, the normalized thermal expansion coefficients are given by $\alpha_{1} / \alpha_{3}=1.5$ and $\alpha_{2} / \alpha_{3}=3$. The volume fractions are given by $C_{1}+C_{2}=0.35$ and $C_{1}=0.88\left(C_{1}+C_{2}\right)$.

Consequently, $F$ may be represented analogously to expression (71) in the form

$$
F=\frac{1}{2} \boldsymbol{\sigma}: \boldsymbol{\epsilon}+\frac{1}{2} \boldsymbol{\Gamma}: \boldsymbol{\epsilon} \theta-\frac{1}{2} C_{v} \theta^{2} .
$$

From Rosen (1970) we know that the average free energy density may be expressed in the following fashion

$$
\bar{F}=\frac{1}{2} \overline{\boldsymbol{\sigma}}: \overline{\boldsymbol{\epsilon}}+\frac{1}{2} \Gamma^{\mathrm{eff}}: \overline{\boldsymbol{\epsilon}} \theta^{0}-\frac{1}{2} C_{v}^{\mathrm{eff}} \theta^{2} .
$$

where $\bar{f}=\frac{1}{V} \iiint_{V} f \mathrm{~d} V$ and where $C_{v}^{\text {eff }}$ is the unknown effective specific heat.

Let $F_{\text {comp }}$ be the free energy calculated over the composite configuration described in Fig. 5 and let $F_{0}$ be the free energy defined in a body identical with the one just specified, except that the inclusion is replaced by the homogeneous material characterized by $\left(\mu^{\text {eff }}, k^{\text {eff }}, \alpha^{\text {eff }}, C_{v}^{\text {eff }}\right)$.

Accounting for the boundary conditions $\vec{T}_{0}=\overrightarrow{0}$ at infinity we find:

$$
\lim _{V \rightarrow \infty} \frac{1}{2} \iiint_{V} \boldsymbol{\sigma}: \boldsymbol{\epsilon}=\lim _{V \rightarrow \infty} \iint_{\partial V} \vec{u} \cdot \vec{T}_{0} \mathrm{~d} S=0,
$$

and $F_{\text {comp }}$ and $F_{0}$ are then expressed as

$$
F_{\text {comp }}=\frac{1}{2} \iiint_{V}\left(\boldsymbol{\Gamma}: \boldsymbol{\epsilon} \theta_{0}-C_{v} \theta_{0}^{2}\right) \mathrm{d} V
$$

and 
(a)

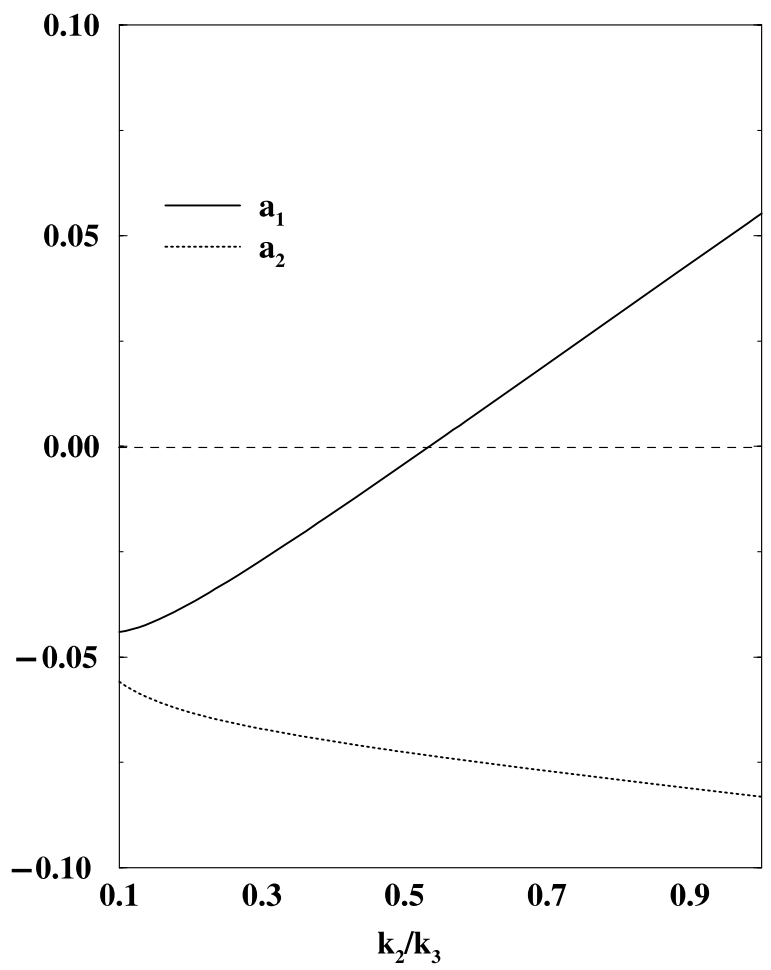

(b)

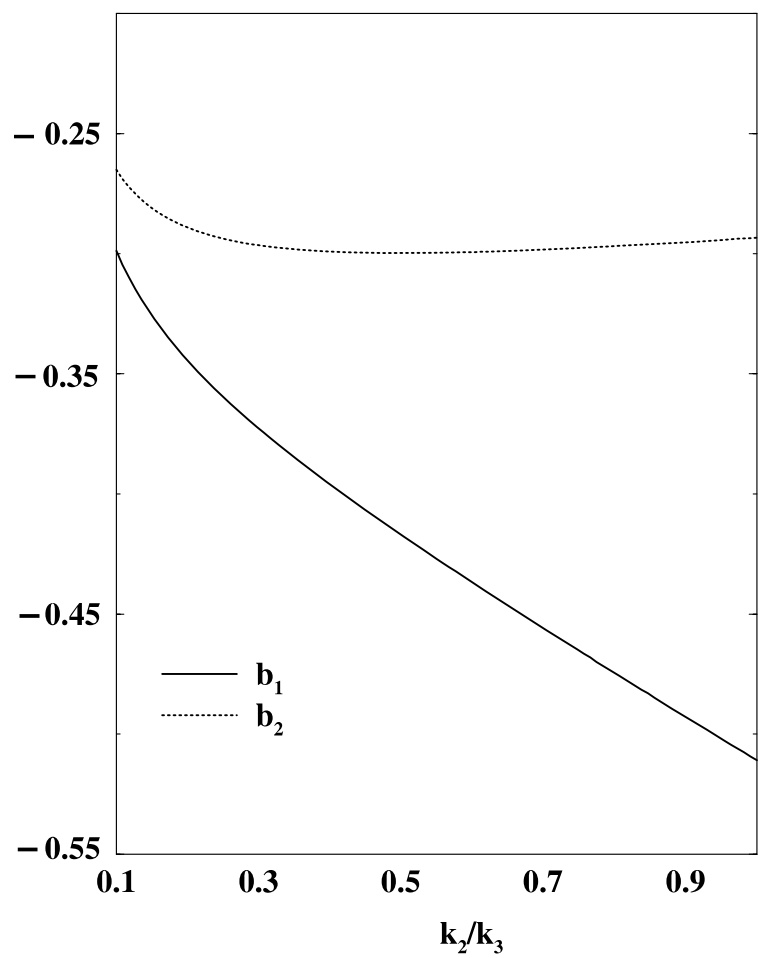

Fig. 4. Normalized radial stress $\sigma_{r r} / \theta_{0} k_{3} \alpha_{3}$ along the interface inclusion/coated-phase $\left(r=R_{1}\right)$ and along the interface coated-phase/ matrix here $\left(r=R_{2}\right)$ of the coated phase (phase 2) of a composite material made of coated inclusions versus the normalized bulk modulus $\left(k_{2} / k_{3}\right)$ of the coated phase and subjected to an uniform temperature rise $\theta_{0}$. $\left(\sigma_{r r}\left(R_{1}\right) / \theta_{0} k_{3} \alpha_{3}=a_{1} \alpha_{2} / \alpha_{3}+b_{1}\right.$ and $\left.\sigma_{r r}\left(R_{2}\right) / \theta_{0} k_{3} \alpha_{3}=a_{2} \alpha_{2} / \alpha_{3}+b_{2}\right)$. The slopes $\left(a_{1}\right.$ and $\left.a_{2}\right)$ are reported in Fig. 4 (a) and the values of $\sigma_{r r} / \theta_{0} k_{3} \alpha_{3}$ for $\alpha_{2} / \alpha_{3}=0$ are reported in Fig. 4(b). $k_{1} / k_{3}=2$. The Young moduli in the different phases are $v_{1}=0.17, v_{2}=v_{3}=0.3$, the normalized thermal expansion coefficient in phase 1 is $\alpha_{1} / \alpha_{3}=1.5$ and the volume fractions are given by $C_{1}+C_{2}=0.35$ and $C_{1}=0.88\left(C_{1}+C_{2}\right)$.

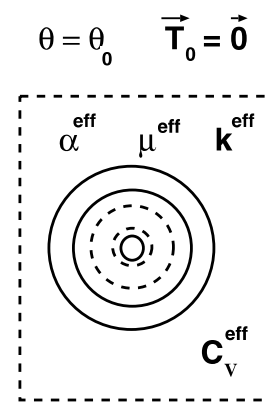

$$
\theta=\theta_{0} \quad \overrightarrow{\mathbf{T}}_{0}=\overrightarrow{0}
$$
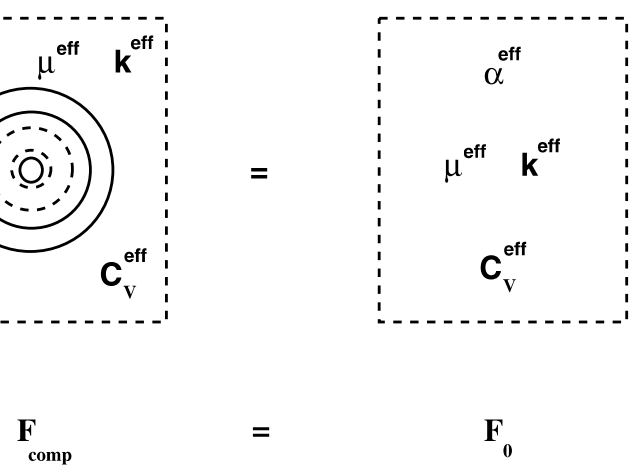

$=$

$\mathbf{F}_{\mathbf{0}}$

Fig. 5. Self-consistent energy condition. 


$$
F_{0}=\frac{1}{2} \iiint_{V}\left(\Gamma^{\mathrm{eff}}: \boldsymbol{\epsilon}^{0} \theta_{0}-C_{v}^{\mathrm{eff}} \theta_{0}^{2}\right) \mathrm{d} V
$$

with $\boldsymbol{\epsilon}^{0}=\boldsymbol{\alpha}^{\text {eff }} \theta_{0}$.

It is worth noticing that $F_{\text {comp }}-F_{0}$ can be split into two parts

$$
F_{\text {comp }}-F_{0}=\frac{1}{2} \iiint_{V}\left(\boldsymbol{\Gamma}: \boldsymbol{\epsilon}-\boldsymbol{\Gamma}^{\mathrm{eff}}: \boldsymbol{\epsilon}^{0}\right) \theta_{0} \mathrm{~d} V+\frac{1}{2} \iiint_{V}\left(C_{v}^{\mathrm{eff}}-C_{v}\right) \theta_{0}^{2} \mathrm{~d} V .
$$

The isotropy of all materials implies

$$
\boldsymbol{\Gamma}: \boldsymbol{\epsilon}=\Gamma_{i j} \epsilon_{i j}=-C_{i j k l} \alpha_{k l} \epsilon_{i j}=-3 k \alpha \operatorname{Tr} \boldsymbol{\epsilon} .
$$

Thanks to Eq. (40), $\operatorname{Tr} \epsilon$ is given in phase $(i)$ by

$$
\operatorname{Tr} \epsilon=3 F_{i}
$$

and consequently

$$
\frac{1}{2} \iiint_{V}\left(\boldsymbol{\Gamma}: \boldsymbol{\epsilon}-\boldsymbol{\Gamma}^{\mathrm{eff}}: \boldsymbol{\epsilon}^{0}\right) \theta_{0} \mathrm{~d} V=\frac{9}{2} \sum_{i=1}^{n+1} \iiint_{V_{i}}\left(k^{\mathrm{eff}} \alpha^{\text {eff }} F_{n+1}-K_{i} \alpha_{i} F_{i}\right) \theta_{0} \mathrm{~d} V .
$$

Given that $k_{n+1}=k^{\mathrm{eff}}, \alpha_{n+1}=\alpha^{\mathrm{eff}}$ and $C_{v}=C_{v}^{\mathrm{eff}}$ in the matrix around the composite inclusion, $F_{\text {comp }}-F_{0}$ finally becomes

$$
F_{\text {comp }}-F_{0}=\frac{1}{2} \sum_{i=1}^{n} \iiint_{V_{i}}\left\{9\left[k^{\text {eff }} \alpha^{\text {eff }} F_{n+1}-K_{i} \alpha_{i} F_{i}\right] \theta_{0}+\left[\left(C_{v}^{\text {eff }}-C_{v_{i}}\right) \theta_{0}^{2}\right]\right\} \mathrm{d} V .
$$

Our criterion for determining the effective specific heat $C_{v}^{\text {eff }}$ is to set

$$
F_{\text {comp }}-F_{0}=0 .
$$

It turns out that $C_{v}^{\text {eff }}$ is then completely determined and the combination of Eqs. (83) and (84) yields

$$
C_{v}^{\mathrm{eff}}=\left\langle C_{v}\right\rangle_{V_{\mathrm{I}}}-\frac{9}{\theta_{0}}\left[k^{\mathrm{eff}} \alpha^{\mathrm{eff}} F_{n+1}-\sum_{i=1}^{n} K_{i} \alpha_{i} F_{i}\right] .
$$

Substituting Eqs. (52) and (51) into Eq. (85) and accounting for $\alpha_{n+1}=\alpha^{\text {eff }}$ provides

$$
C_{v}^{\mathrm{eff}}=\left\langle C_{v}\right\rangle_{V_{\mathrm{I}}}-9\left\{k^{\mathrm{eff}}\left(\alpha^{\mathrm{eff}}\right)^{2}-\sum_{i=1}^{n} \frac{c_{i} k_{i} \alpha_{i}}{Q_{11}^{(n)}}\left[\left(\alpha^{\mathrm{eff}}-M_{1}^{(n+1)}\right) Q_{11}^{(i-1)}+M_{1}^{(i)} Q_{11}^{(n)}\right]\right\},
$$

and it follows from Eq. (69) that $C_{v}^{\text {eff }}$ can be put finally in the form

$$
C_{v}^{\mathrm{eff}}=\left\langle C_{v}\right\rangle_{V_{\mathrm{I}}}-9\left\{k^{\mathrm{eff}} \alpha^{\mathrm{eff}} M_{1}^{(n+1)}-\sum_{i=1}^{n} c_{i} k_{i} \alpha_{i} M_{1}^{(i)}\right\} .
$$

Note that, as for the thermal conductivity and for the thermal expansion coefficient, if $C_{v_{(i)}}^{\text {eff }}$ denotes the effective specific heat associated with a $(i+1)$-phase model, we get the following relation between $C_{v_{(n)}}^{\text {eff }}$ and $C_{v_{(n-1)}}^{\text {eff }}$

$$
C_{v_{(n)}}^{\mathrm{eff}}=\frac{R_{n-1}^{3}}{R_{n}^{3}} C_{v_{(n-1)}}^{\mathrm{eff}}+\left(1-\frac{R_{n-1}^{3}}{R_{n}^{3}}\right) C_{v_{n}}-\frac{27 \frac{R_{n-1}^{3}}{R_{n}^{3}}\left(\frac{R_{n-1}^{3}}{R_{n}^{3}}-1\right)\left(k_{(n-1)}^{\mathrm{eff}} \alpha_{(n-1)}^{\mathrm{eff}}-k_{n} \alpha_{n}\right)^{2}}{3 k_{n}+4 \mu_{n}+3\left(1-\frac{R_{n-1}^{3}}{R_{n}^{3}}\right)\left(k_{(n-1)}^{\mathrm{eff}}-k_{n}\right)} .
$$

If $n=2$, this equation with $\left(C_{v_{(1)}}^{\text {eff }}=C_{v_{1}}\right)$ provides the effective specific heat which is given by (Stolz, 1999) for a two-phase CSA (with a different sign in the definition of $C_{v}$ ). 
Let $T_{0} C_{p} / \rho$ be the specific heat per unit volume at constant stress. The effective specific heats ( $C_{v}^{\text {eff }}$ and $C_{p}^{\text {eff }}$ ) are related (Rosen, 1970; Christensen, 1979) by the following expression

$$
\mathbf{C}_{p}^{\mathrm{eff}}-\mathbf{C}_{v}^{\mathrm{eff}}=\boldsymbol{\alpha}^{\mathrm{eff}}: \mathbf{C}^{\mathrm{eff}}: \boldsymbol{\alpha}^{\mathrm{eff}}
$$

and, since we consider isotropic behaviours, the above-mentioned relation becomes

$$
C_{p}^{\mathrm{eff}}-C_{v}^{\mathrm{eff}}=9 k^{\mathrm{eff}}\left(\alpha^{\mathrm{eff}}\right)^{2} .
$$

\section{Results and conclusion}

The aim of this paper was to extend the field of possible application of the $(n+1)$-phase model to multiply coated inclusion-reinforced composite having a thermal or a thermoelastic behaviour. In order to prove the tractability of these models, illustrative examples of the influence of an interphase lying between the particles and the matrix of a particle-reinforced composite material are given in Figs. 6-8. In the first and third case the volume fraction of the coating phase is varying from 0 up to the total initial volume fraction of the particles (for different values of the interphase conductivity coefficient in the first case and for different values of the thermal expansion coefficient in the third case). In the second case the volume fraction of the coating and particle phases are fixed but both the thermal expansion coefficient and the bulk modulus of the coating phase are varying.

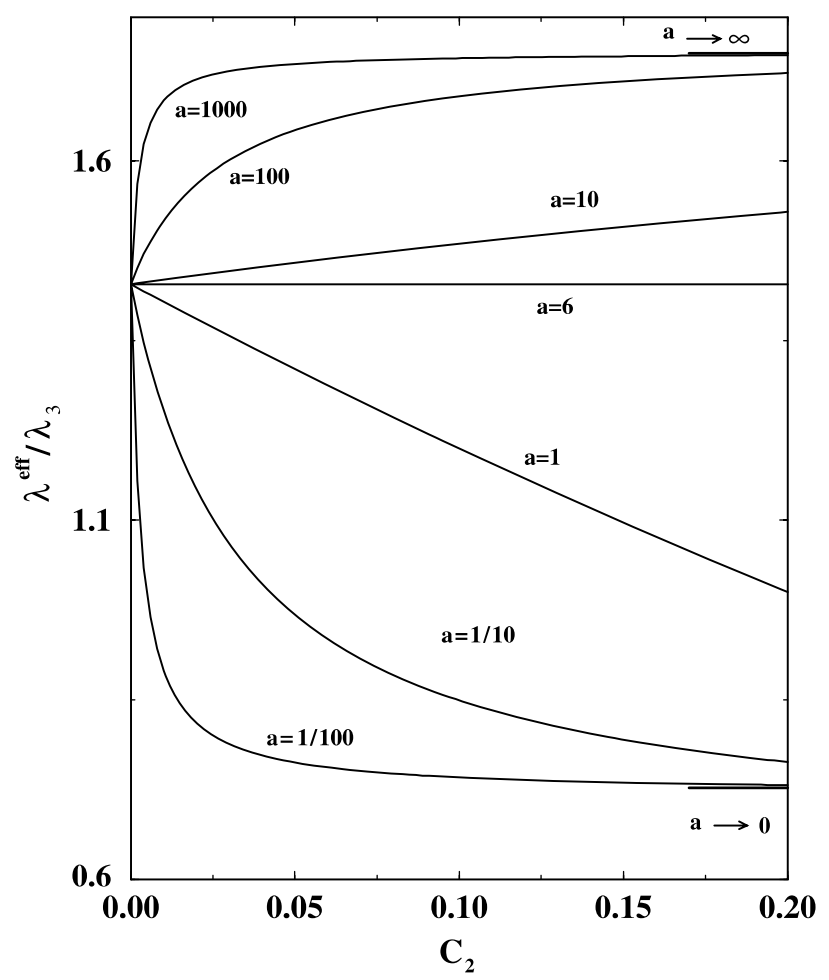

Fig. 6. Normalized effective conductivity coefficient $\lambda^{\text {eff }} / \lambda_{3}$ of a composite material, made of coated inclusions versus the volume fraction $\left(C_{2}\right)$ of the coated phase (phase 2) for different values of $a=\lambda_{2} / \lambda_{3}$ ( 1 denotes the inclusion and 3 the matrix). $\lambda_{1} / \lambda_{3}=6$, $C_{1}+C_{2}=0.2$. 


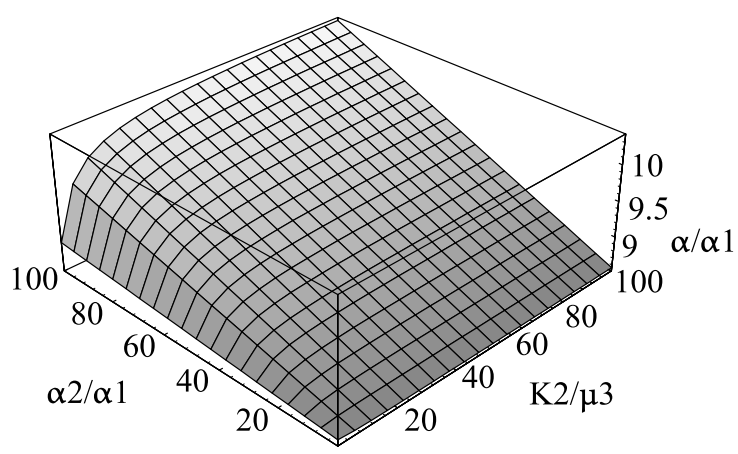

Fig. 7. Normalized effective thermal expansion coefficient $\alpha / \alpha_{1}$ of a composite material, made of coated inclusions. The volume fraction of the inclusion (phase 1) and of the coated phase (phase 2) are respectively 0.1 and 0.01 and the elastic behaviours of the different phases are characterized by $k_{1} / \mu_{3}=15, \mu_{1} / \mu_{3}=7, k_{3} / \mu_{3}=2, \mu_{2} / \mu_{3}=4, \alpha_{3} / \alpha_{1}=10$.

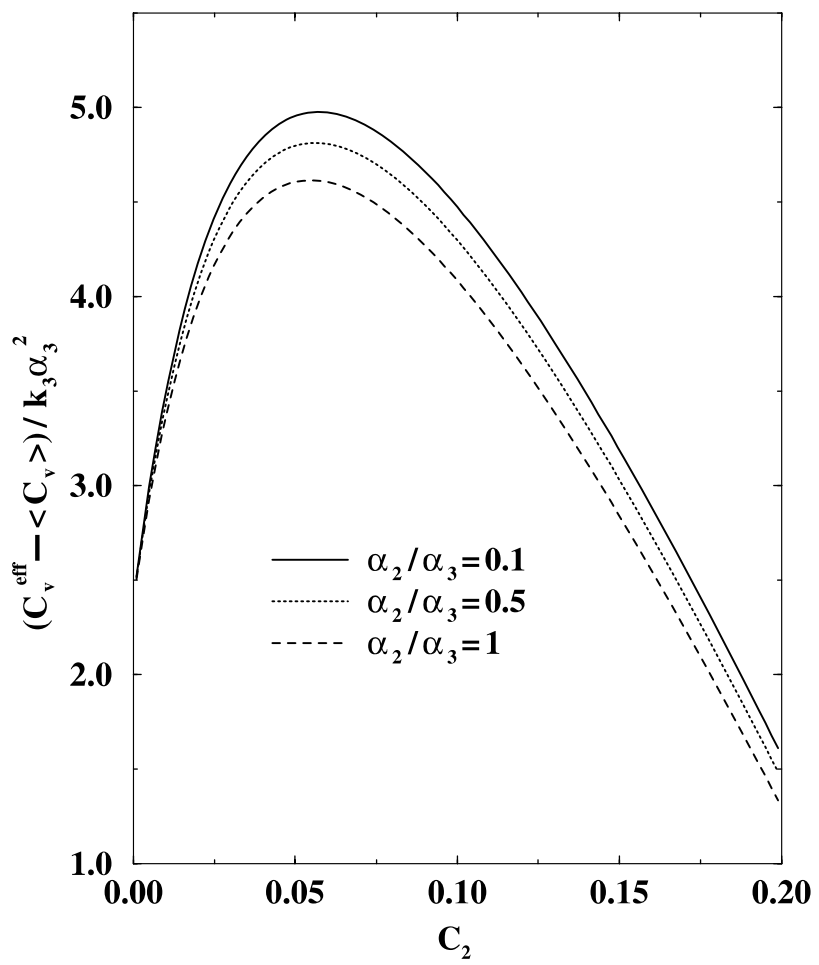

Fig. 8. Normalized difference of the effective specific heat with the average of the specific heats of each phase of a composite material, made of coated inclusions versus the volume fraction $\left(C_{2}\right)$ of the coated phase (phase 2) for different values of $\alpha_{2} / \alpha_{3}(3$ denotes the matrix). $k_{1} / k_{3}=2, k_{2} / k_{3}=0.1, v_{1}=0.17, v_{2}=v_{3}=0.3, \alpha_{1} / \alpha_{3}=1.5, C_{1}+C_{2}=0.2$.

These results can also be used to determine the overall behaviour of material with gradient of properties by discretization as already done in Hervé and Zaoui (1995a) in order to predict the effective behaviour of fibre-reinforced composite having an accommodating interphase. 
It is worth noting that the form of these results allows to use a recursive algorithm to determine the effective thermal conductivity, the effective thermal expansion coefficient and the effective specific heats of a composite material according to the $(n+1)$-phase model.

Moreover, Figs. 3 and 4 prove that all these results are very useful to determine some major local parameters such as the radial stress along the interfaces of a coated phase around the inclusion. The problem of debonding along the interfaces of the coated phase is often meet in the case of fibre-reinforced composites. The extension of such a study to multiply coated fibre-reinforced composites should bring interesting answers to a lot of practical problems.

\section{Acknowledgement}

I am indebted to Claude Stolz for fruitful discussions.

\section{References}

Alberola, N.D., Benzarti, K., 1997. Meso- et microstructures des composites unidirectionnels: modélisation du comportement viscoélastique. Comptes Rendus à l'Académie des Sciences 325 (Série II), 249-255.

Alberola, N.D., Benzarti, K., 1998. Modeling of the relationship between morphology and viscoelastic behavior of unidirectional fiberreinforced polymers. Polymer Engineering and Science 38 (3), 429-438.

Alberola, N.D., Mele, P., 1994. Composites particulaires: modélisation du comportement viscoélastique, assortie du concept de percolation. Comptes Rendus à l'Académie des Sciences 319 (Série II), 1129-1134.

Alberola, N.D., Mele, P., 1997. Interface and mechanical coupling effects in model particulate composite. Polymer Engineering and Science, 37, 1712-1721.

Beurthey, S., 2000. Structural morphology and relaxation spectra of viscoelastic heterogeneous materials. European Journal of Mechanics A: Solids 19, 1-16.

Bornert, M., Hervé, E., Stolz, C., Zaoui, A., 1993. Modelling the strain heterogeneities in a two-phase elastoplastic material. In: Proceedings of Mecamat 93 International Seminar on Micromechanics of Materials, Moret-sur-Loing, France, pp. $26-37$.

Bornert, M., Hervé, E., Stolz, C., Zaoui, A., 1994. Self-Consistent approaches and strain heterogeneities in two-phase elastoplastic materials. Applied Mechanics Review 47 (1), 66-76.

Christensen, R.M., 1979. Mechanics of Composite Materials. Wiley Interscience.

Fen-Chong, T., Hervé, E., Zaoui, A., 1999. Micromechanical modelling of intracellular pressure-induced viscoelastic shrinkage of foams: application to expanded polystyrene. European Journal of Mechanics 18, 201-218.

Hashin, Z., 1972. NASA contractor report (NASA-CR-1974).

Hashin, Z., Shtrikman, S., 1962. A variational approach to the theory of the effective magnetic permeability of multiphase materials. Journal of Applied Physics 33 (10), 3125-3131.

Hervé, E., Dendievel, R., Bonnet, G., 1995. Steady-state power-law creep in “inclusion matrix" composite materials. Acta Metallurgica et Materialia 43, 4027-4034.

Hervé, E., Pellegrini, O., 1995. The elastic constants of a material containing spherical coated holes. Archives of Mechanics 47 (2), 223-246.

Hervé, E., Stolz, C., Zaoui, A., 1993. Morphological representative pattern-based micromechanical modelling. In: Mecamat 93 International Seminar on Micromechanics of Materials, Moret-sur-Loing, France, pp. 3-13.

Hervé, E., Zaoui, A., 1993. n-layered inclusion-based micromechanical modelling. International of Engineering and Sciences 31 (1), $1-10$.

Hervé, E., Zaoui, A., 1995a. Elastic behaviour of multiply coated fibre-reinforced composites. International of Engineering and Sciences 33 (10), 1419-1433.

Hervé, E., Zaoui, A., 1995b. Morphological n-layered cylindrical pattern-based micromechanical modelling. In: IUTAM Symposium on Anisotropy, Inhomogeneity and Nonlinearity in Solid Mechanics, pp. 403-408.

Levin, V.M., 1968. On the coefficient of thermal expansion of heterogeneous materials. Mekhanika Tverdogo Tela 88 (in Russian).

Rosen, B.W., 1970. Thermoelastic energy functions and minimum energy principles for composite materials. International of Engineering and Sciences 8, 5-18.

Rosen, B.W., Hashin, Z., 1970. Effective thermal expansion coefficients and specific heats of composite materials. International of Engineering and Sciences 8, 157-173. 
Schapery, R.A., 1968. Thermal expansion coefficients of composite materials based on energy principles. Journal of Composite Materials 2 (3), 380-404.

Stolz, C., 1999. Approche variationnelle du comportement des milieux hétérogènes en thermoélasticité linéaire. Comptes Rendus à l'Académie des Sciences 327 (Série II), 185-192.

Thébaud, F., Michel, J.C., Hervé, E., Suquet, P., Zaoui, A., 1992. Modelling nonlinear behavior of composites with imperfect interfaces. In: Proceedings of the 13th Risø International Symposium on Materials Science, pp. 467-472. 